Journal of Nanoparticle Research, 15:1823 (2013)

DOI: 10.1007/s11051-013-1823-y

\title{
Zigzag-shaped nickel nanowires via organometallic template-free route
}

$\underline{\text { Meital Shviro, András Paszternák, }} \underline{\text { Avraham Chelly, David Zitoun }}$ 


\title{
Zigzag-shaped nickel nanowires via organometallic template-free route Meital Shviro ${ }^{\S, 1}$, András Paszternák ${ }^{\S, 1}$, Avraham Chelly ${ }^{2}$ and David Zitoun ${ }^{1, *}$ \\ ${ }^{1}$ Bar Ilan University, Department of Chemistry \\ ${ }^{2}$ Bar Ilan University, Department of Engineering \\ Bar Ilan Institute of Nanotechnology and Advanced Materials (BINA) \\ Ramat Gan 52900, ISRAEL
}

\begin{abstract}
In this manuscript, the formation of nickel nanowires (average size: several tens to hundreds of $\mu \mathrm{m}$ long and 1.0-1.5 $\mu \mathrm{m}$ wide) at low temperature is found to be driven by dewetting of liquid organometallic precursors during spin coating process and by self-assembly of Ni clusters. Elaboration of metallic thin films by low temperature deposition technique makes the preparation process compatible with most of the substrates. The use of iron and cobalt precursor shows that the process could be extended to other metallic systems. In this work, AFM and SEM are used to follow the assembly of Ni clusters into straight or zigzag lines. The formation of zigzag structure is specific to the Ni precursor at appropriate preparation parameters. This template free process allows a control of anisotropic structures with homogeneous sizes and angles on standard $\mathrm{Si} / \mathrm{SiO}_{2}$ surface.
\end{abstract}

KEYWORDS: electroless deposition, nickel, nanowires, coating, magnetic

\footnotetext{
$\S$ These authors equally contribute to the work.
} 


\section{INTRODUCTION}

Ni thin coatings and nanowires can be obtained by organometallic wet deposition, (Shviro, M.; Zitoun, D., 2012) the focus of the present study is to understand the formation of metallic lines including nanowires with specific zigzag shape, Co and NiFe lines. Metallic zigzag lines are used for different applications in nanoelectronic devices; (He, et al., 2007) as reported for devices like highly stretchable conductive electrodes, (Hyun, et al., 2011) anti-Stokes emission testers (Kim, et al., 2008) or data storage with NiFe zigzag lines (Yu, et al., 2003).

On the one hand, wet deposition by spin coating is a classical process for material deposition. In most of the cases, this deposition technique aims at controlling thickness of molecular, polymeric or nanoparticles films. Wetting and dewetting are critical events for homogeneous deposition which depend on the surface energy (substrate/solution) (Xia, et al., 2001) (Müller \& Spolenak, 2010) (Ye \& Thompson, 2011). Heterogeneous deposition is usually seen as a failure of the process. Nevertheless, in some cases, the heterogeneous deposition can be used as a template free directed deposition. For instance, coffee ring effect is a well-known drying process which drives particles on the edge of hemispherical droplets, resulting in the formation of circles of particles (Deegan, et al., 1997) (Deegan, 2000). This phenomenon has been observed during the deposition of nanoscale and microscale particles (Choi, et al., 2010). Very interestingly, coffee ring effect could also bring some benefit to deposit connected rings. Drying process could also direct the deposition of self-assembled nanostructures (Zhang, et al., 2002).

On the other hand, even metallic thin films are rarely stable structures, but are instead kinetically frozen as a consequence of formation far from equilibrium. Therefore, when films are heated so that atomic diffusion occurs, they often dewet to form arrays of islands. The rate of dewetting is higher in thinner films, so that the temperature at which dewetting occurs decreases with decreasing film thickness (Ye \& Thompson, 2011). Thin films of $\mathrm{Ni}$ dewets on $\mathrm{SiO}_{2}$ during annealing in the solid state (Petersen \& Mayr, 2008). Ni exhibits the common capillarity driven dewetting mechanism of void nucleation and growth during annealing. In situ studies reveal the isotropic dewetting of Ni-films deposited on amorphous $\mathrm{SiO}_{2}$ (Felici, R.; Jeutter, N. M.; Mussi, V.; Buatier de Mongeot, F.; Boragno, C.; Valbusa, U.; Toma, A.; Zhang, Y. W.; Rau, C.; Robinson, I. K., 2007). Hole formation is initiated at temperatures below $100{ }^{\circ} \mathrm{C}$, in $5 \mathrm{~nm}$ thin layers. On a ripple patterned surface dewetting reveals a different kinetics as on the smooth substrate. Due to an additional driving force of curvature induced diffusion, large voids are formed already at low annealing temperatures, forming nanorods. 
In this research, we report on a deposition process where solution dewetting and metal dewetting interplay to form anisotropic structures grown on amorphous $\mathrm{SiO}_{2}$ layer. The formation of metallic lines arises from a very specific sequence: (i) reaction of organometallic complex, (ii) evaporation of the solvent and by product, (iii) surface diffusion of metal clusters. The self-assembly of $\mathrm{Ni}, \mathrm{Co}$ and $\mathrm{NiFe}$ clusters into lines is investigated by AFM and SEM, the line shape is correlated to the film process parameters.

\section{EXPERIMENTAL PROCEDURE}

$\mathrm{Ni}(\mathrm{COD})_{2}(\mathrm{STREM}, 98 \%), \mathrm{Co}_{2}(\mathrm{CO})_{8}$ (Acros, 95\%) and $\mathrm{Fe}(\mathrm{CO})_{5}$ (Acros, 99.5\%) were stored at $-20{ }^{\circ} \mathrm{C}$ in a nitrogen filled glovebox $\left(\mathrm{H}_{2} \mathrm{O}<0.1 \mathrm{ppm}, \mathrm{O}_{2}<2 \mathrm{ppm}\right)$. Mesitylene (1,3,5-trimethylbenzene, Acros, 97\%) was dried, degassed and stored on a molecular sieve. Pieces of silicon wafer (Virginia semiconductors, $\langle 100\rangle$, n-type, 4-6 $\Omega . \mathrm{cm}$ ) were washed according to standard procedure. A solution of 0.03 mol. $\mathrm{L}^{-1}$ of $\mathrm{Ni}(\mathrm{COD})_{2}$ in mesitylene was used to coat the surface of Si pieces of $5 * 5 \mathrm{~mm}^{2}$. In a typical experiment, the solution was spin-coated in a glovebox at different speeds (2000, 3000, 4000 and 6000 $\mathrm{rpm}$ ) for $1 \mathrm{~min}$. The coating was then annealed for 10 minutes on a heating plate or in an oven at a constant temperature ranging from $80^{\circ} \mathrm{C}$ up to $150^{\circ} \mathrm{C}$ in inert atmosphere.

The morphology of the coating was studied using an Atomic Force Microscope (AFM, ICON, Bruker) in tapping mode with a silicon nitride tip (FESP) and a Scanning Electron Microscope (Inspect, FEI, 3-10 $\mathrm{kV}$ accelerating voltage).

\section{RESULTS AND DISCUSSIONS}

\subsection{Parallel lines and pathway to complex metallic networks}

Large scale uniform line pattern can be created on the whole substrate surface using metallic precursor and spin coating technique (Fig. 1A). The process is based on the thermal decomposition of Ni ( $\eta^{4}$ $\left.\mathrm{C}_{8} \mathrm{H}_{12}\right)_{2}$ at temperature as low as $80{ }^{\circ} \mathrm{C}$. The Ni-complex has been dissolved in mesitylene and spin coated on a substrate, heated on a hotplate to decompose the organometallic precursor and evaporate solvent and by-product. The heating step has also been carried in an oven instead of a heating plate to get more homogeneous temperature on the sample. The results obtained in both cases do not show a significant improvement using the oven. The process results in the formation of metallic Ni line as shown EDAX analysis (Fig. S1). Ni crystallizes in the face-centered cubic (FCC) phase with lattice parameters corresponding to bulk Ni (Fm-3m, a = 3.52 $\mathrm{A}$ ) (JCPDS-04-0850) as shown from X-ray diffraction pattern (Fig. S2). A small amount of bunsenite (NiO) is observed as a result of air exposure of the sample after synthesis. The sample does not display any noticeable oxidation after a few weeks of air exposure, suggesting the formation of an efficient passivation layer (Sikora \& Macdonal, 2002). The magnetization 
are measured following a zero-field-cooled/field-cooled (ZFC/FC) measurement from $2 \mathrm{~K}$ to $300 \mathrm{~K}$ with a low external field $\mu_{0} \mathrm{H}=0.005 \mathrm{~T}$ (Fig. S3). The sample displays a superparamagnetic behavior with a ZFC maximum at $\mathrm{T}=160 \mathrm{~K}$. Assuming this maximum would be close to the average blocking temperature and the bulk magnetocrystalline anisotropy value $\mathrm{K}_{\mathrm{V}}=5.10^{3} \mathrm{~J}_{\mathrm{J}} \mathrm{m}^{-3}$, the line would consist of $30 \mathrm{~nm} \mathrm{Ni}$ particles.

The phenomenon of the line-network formation has been discussed as a selective dewetting of the organometallic precursor solution into elongated droplets. The formation of elongated droplets results from the centrifugal force during spin-coating since the lines have been obtained on several substrates (glass, silicon wafers [111] or [100]) only when the precursor has been spincast. The average distance between the lines is reproducible and found to be $4.8 \pm 1.5 \mu \mathrm{m}$ for a sample spin-coated at $3000 \mathrm{rpm}$ and annealed at $80^{\circ} \mathrm{C}$ for $10 \mathrm{~min}$ (Fig. 1A-B). The lines are several tens to hundreds of $\mu \mathrm{m}$ long and $1.0 \pm 0.3$ $\mu \mathrm{m}$ wide.
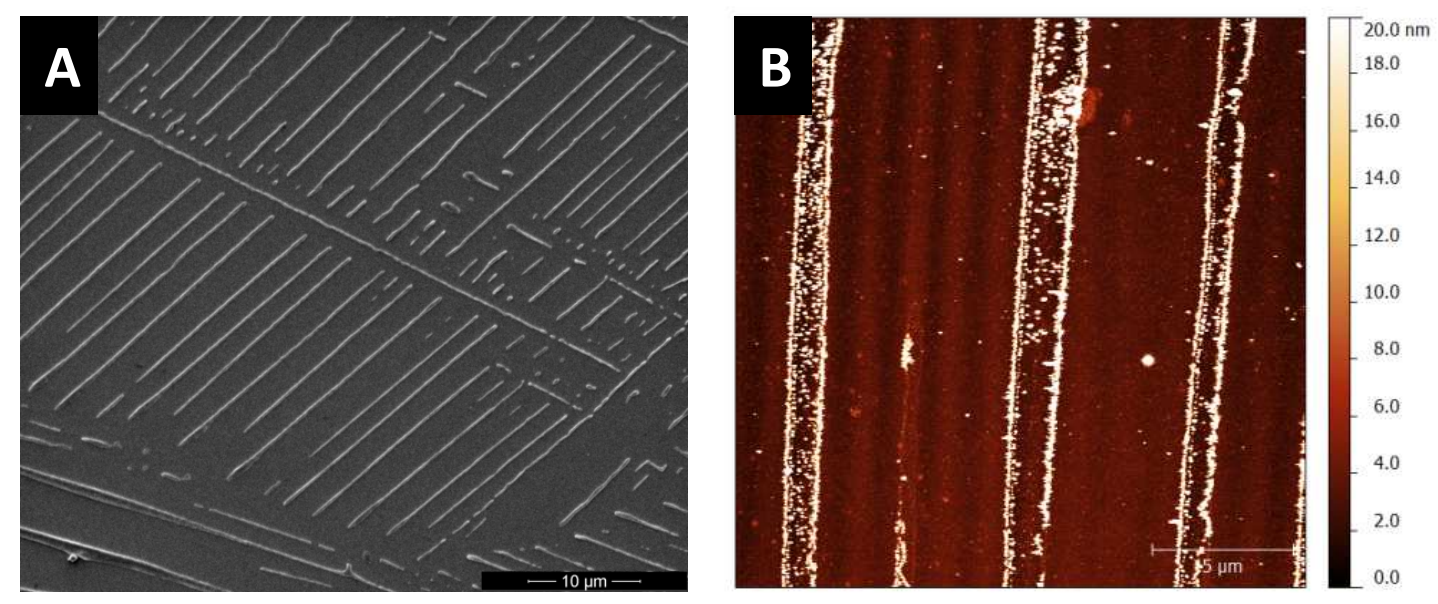

Figure 1: Ni lines with wafer centered on the spin coater at $3000 \mathrm{rpm}$ after thermal decomposition of the precursor at $80^{\circ} \mathrm{C}$ observed by SEM (A) and AFM (B)

While the substrate is centered on the spin coater, the lines grow parallel and several areas of parallel lines with different orientations are observed (Fig. 1A). To achieve parallel lines on a sub-millimeter scale, we have placed the substrate outside the rotation axis of the spin coater and observed that most of the lines are parallel on all the substrate (Fig. 2A).

We explore the potential to create more complex patterns on the substrate with consecutive $\mathrm{Ni}$ depositions. As an example, network structure of metallic lines created by a two-step process is presented on Fig. 2. The off centered Si wafer was coated with Ni precursor at $4000 \mathrm{rpm}$ and annealed at $80^{\circ} \mathrm{C}$ (Fig. 
2A). Then, the same wafer was rotated by $90^{\circ}$ and placed back on the spin coating holder for a second coating. We performed the spin coating and annealing steps in the same conditions on the off centered substrate. Consecutive depositions can effectively create a grid (Fig. 2B and inset). The angles between the lines created during the two successive metal deposition processes are close to $90^{\circ}$ and obviously result from the rotation of the substrate between the two steps.
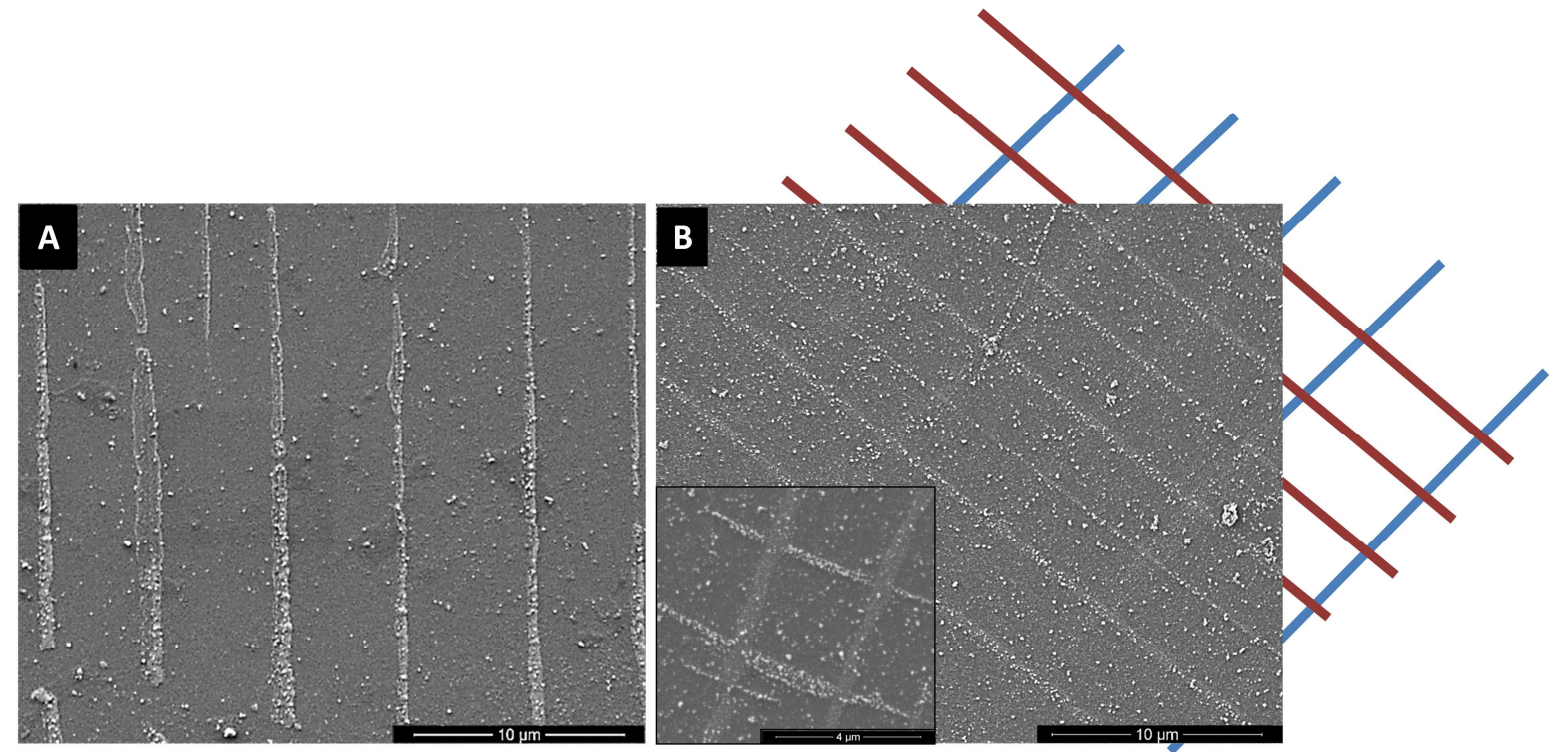

Figure 2: SEM of Ni lines observed on off-centered wafer after first deposition and annealing (A), after second deposition and annealing (wafer rotated $\left.90^{\circ}\right)(\mathrm{B})$

\subsection{Characterization of metallic line-shapes}

The Ni lines do form a network of parallel lines formed of assembled Ni nanoparticles, which means that each line's morphology should be affected by the deposition conditions. The shape and density of the lines should then depend on the spin coating and annealing parameters.

We have investigated the shape of the created metallic lines by varying the speed of the spin coater from $600 \mathrm{rpm}$ to $10000 \mathrm{rpm}$ and the annealing temperature from $50^{\circ} \mathrm{C}$ to $150^{\circ} \mathrm{C}$. The lines are formed only in a narrower window (2000 to $6000 \mathrm{rpm}$ ), while $\mathrm{Ni}$ forms an almost uniform coating for lower speed and isolated particles for higher speed.

By changing the speed of the spin coating process from 2000 to $6000 \mathrm{rpm}$, we observe different line shapes, as shown from AFM images on Figure 3. In the case of lower speed (A: $2000 \mathrm{rpm}, \mathrm{B}: 3000 \mathrm{rpm}$ ), particles form pairs of lines with almost parallel border-shape. At $4000 \mathrm{rpm}$, pairs of Ni-lines display a zigzag shape (Fig. 3C). Applying higher spin coating speed (D: $6000 \mathrm{rpm}$ ), tear shapes particles chains 
are formed. The average diameter of the created lines is between 1.2-1.6 $\mu \mathrm{m}$ (measured between the border-lines of the bands, perpendicular to the band longer axis).
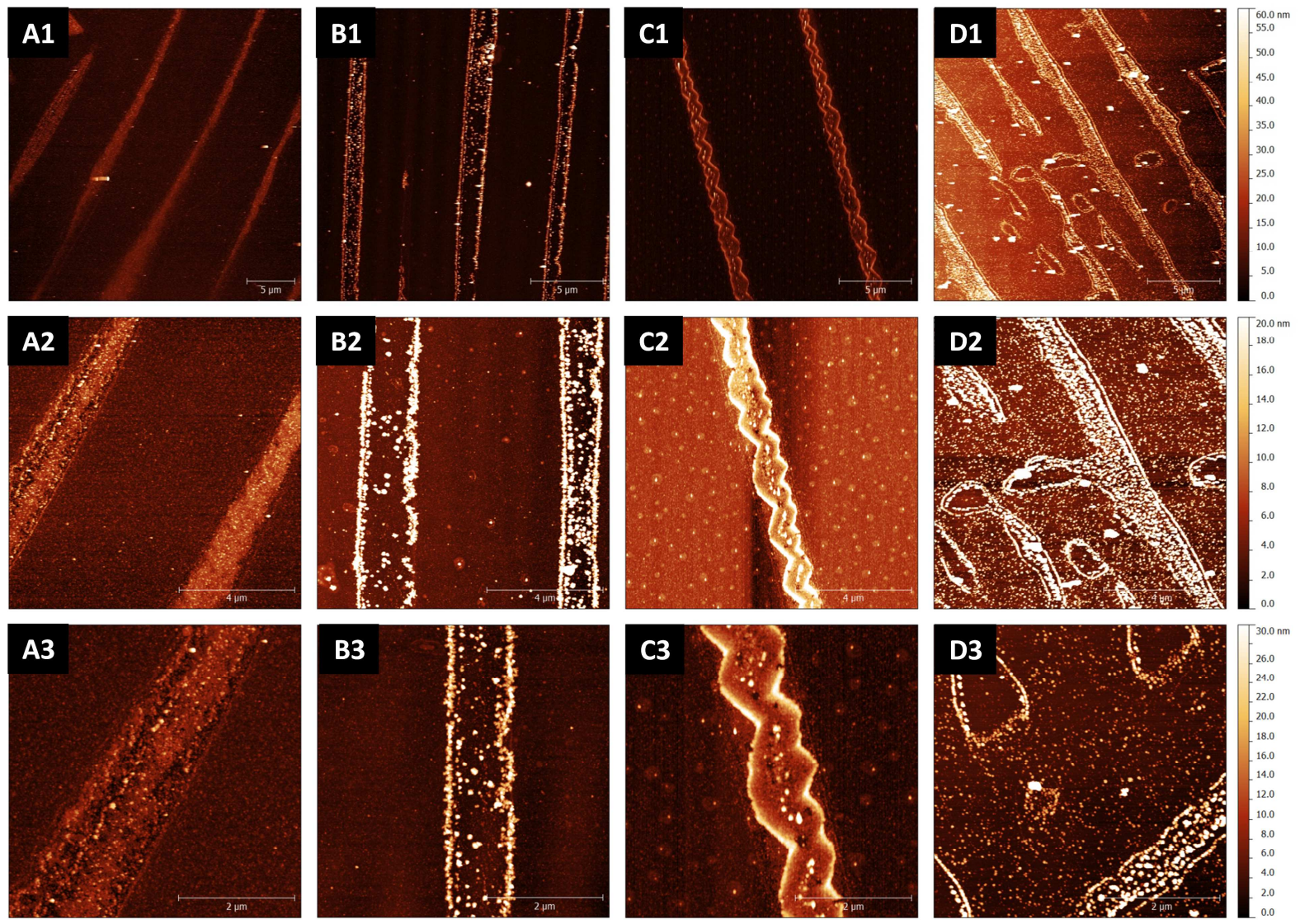

Figure 3: AFM images of $\mathrm{Ni}$ lines - Ni lines density dependence from the spin coating speed $(\boldsymbol{A}-\mathbf{2 0 0 0}$ rpm; $B-3000$ rpm, $C-4000 \mathrm{rpm}, \mathrm{D}-\mathbf{6 0 0 0} \mathrm{rpm}$ ), annealing temperature $80^{\circ} \mathrm{C}$

The annealing step right after the spin coating is crucial to obtain metallic lines. An increasing temperature results in a more homogeneous nucleation on the Si substrate and also in an increase of the density of particles (Fig. 4). Temperature drives the Ni to diffuse and nucleate on all the substrate without a real contrast. Increase of the individual particles size and density was monitored by changing the temperature from $80{ }^{\circ} \mathrm{C}$ to $150{ }^{\circ} \mathrm{C}$. 

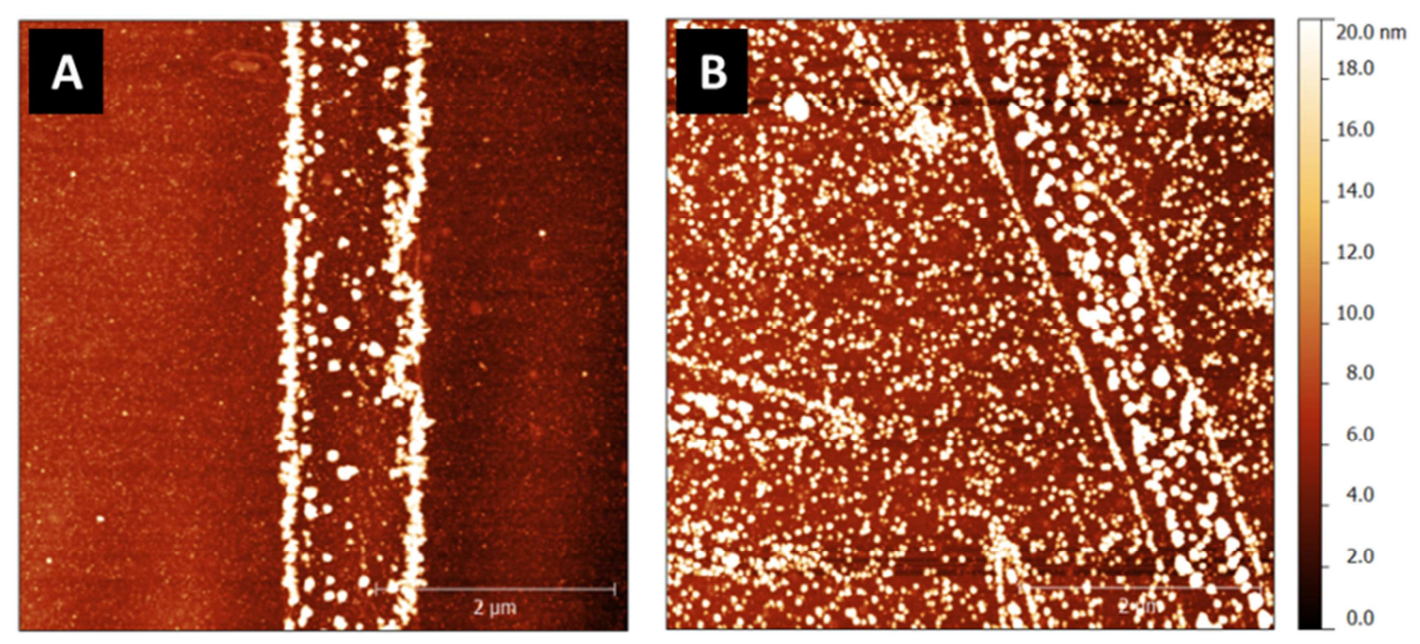

Figure 4: Line formation mechanism (AFM) - Atomic force microscopy $(5 \mu m \times 5 \mu m)$ images of $\mathrm{Ni}$ nanowires formed at spin coating speed 3000 rpm and annealed at $80^{\circ} \mathrm{C}(\boldsymbol{A})$ or $150{ }^{\circ} \mathrm{C}(\boldsymbol{B})$

\subsection{Zigzag lines}

As already observed on Fig.3-C1 and -C2, the Ni clusters adopt a very intriguing zigzag shape when the lines are formed around $4000 \mathrm{rpm}$ and $80{ }^{\circ} \mathrm{C}$. Only under these experimental conditions, we could observe zigzag lines, which precludes the hypothesis of a template effect from the Si substrate. This hypothesis is consistent with the fact that $\mathrm{Si}$ wafer surface displays a native oxide layer which is amorphous. The substrate can be considered as a perfectly flat amorphous solid and has no influence on the nucleation and growth process of Ni clusters. The detailed characterization of zigzag lines has been carried out by AFM. 

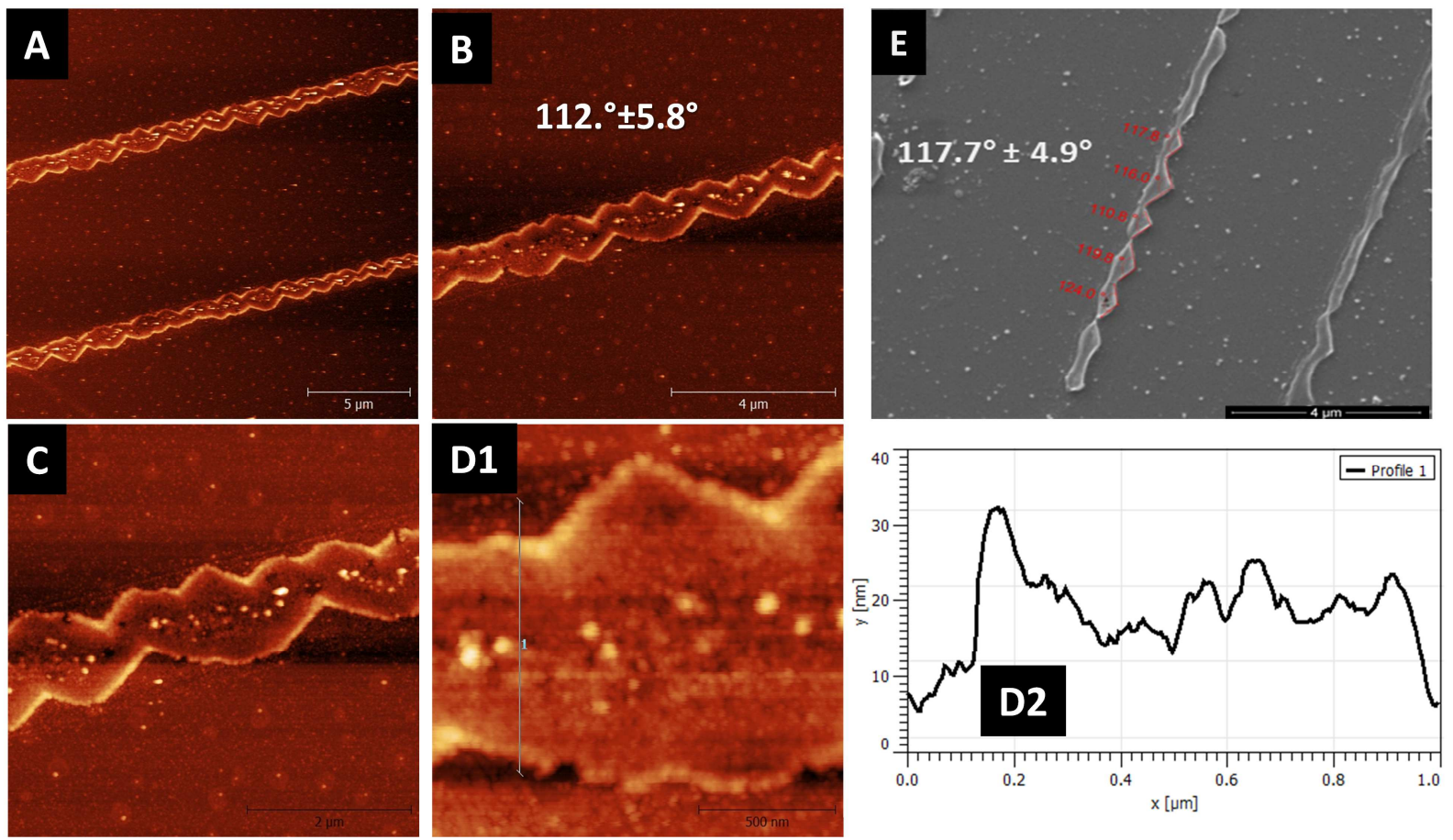

Figure 5: Zigzag lines investigated by SEM (A) and AFM (B)

Pairs of lines grow parallel (Fig. 5A), with a typical length for the zigzag structure of several tens of $\mu \mathrm{m}$ (Fig. 5B.). The thickness of the zigzag band is around $20 \mathrm{~nm}$ at the edges and $10 \mathrm{~nm}$ inside the band (Fig. 5D). The individual nanoparticles, building up the lines are also visible (Fig. 5C-5D).

The process is reproducible and zigzag lines on different samples are displayed on figure 7 . The shape of the lines has been characterized using AFM and SEM. The average value of the angle at the small triangles is $117.7^{\circ} \pm 4.9^{\circ}$ in the case of the sample controlled by SEM and $112.0^{\circ} \pm 5.8^{\circ}$ on another sample checked by AFM. This value is very close to $120^{\circ}(\pi / 3)$ which evocates 3 -fold axis symmetry.

On AFM images, next to the zigzag band, an underlying wider band is also present with average diameter of $1.4 \pm 0.1 \mu \mathrm{m}$ measured between the border-lines of the bands, perpendicular to the band longer axis (Fig. 6A). 

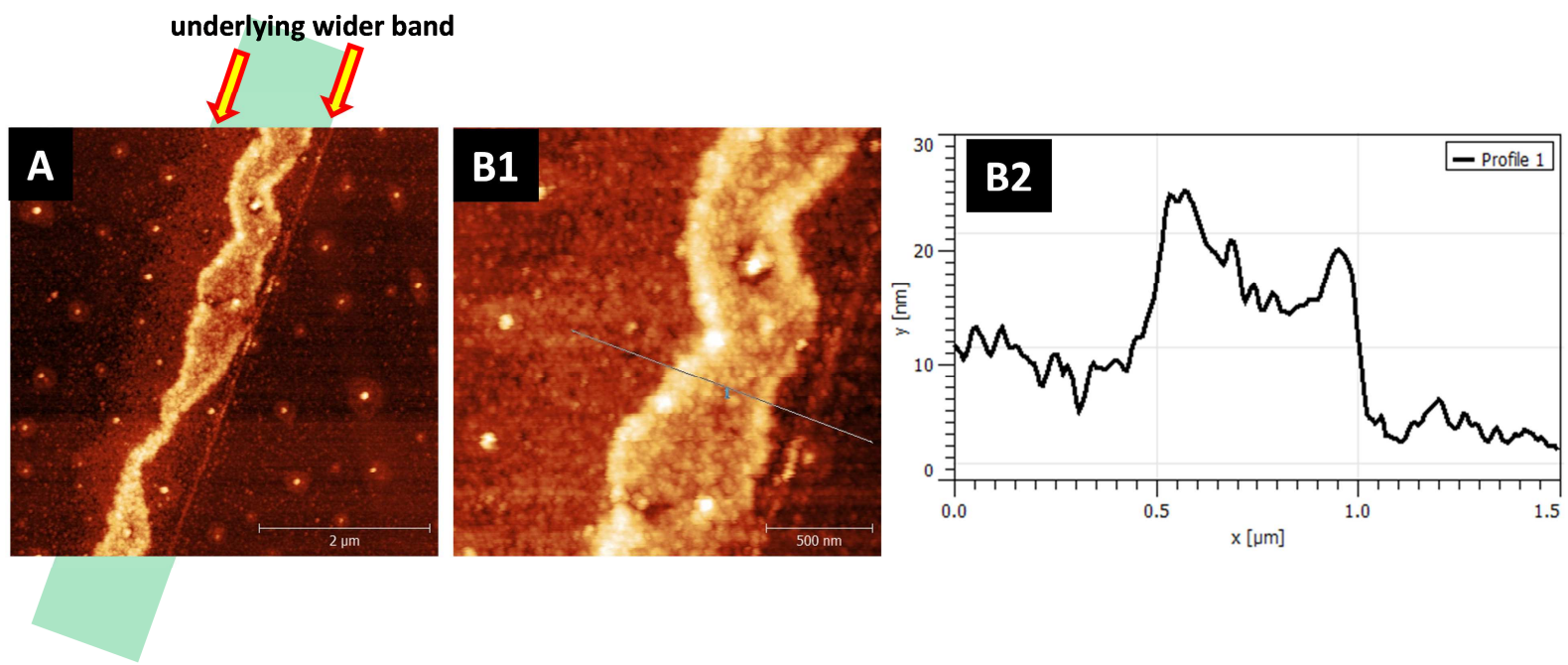

Figure 6: AFM of the underlying wider band and upper zigzag pattern (A), zigzag pattern (B1) and its corresponding line profile (B2)

As advocated above, the Si substrate cannot act as a template since the amorphous native oxide does not allow any oriented crystal growth. This theoretical piece of argument is corroborated by all the experiments done at different speed and temperature which do not lead to a zigzag shape. Therefore, we assume that the shape is induced by the evaporation mechanism and the Ni NPs diffusion according to the following considerations.

\section{I. $\quad$ Thick border line}

The line formation has been studied in our previous articles (Shviro, M.; Zitoun, D., 2012) (Shviro \& Zitoun, 2013). The Ni line results from the formation of elongated radial droplets of wet organometallic species during the spin coating steps. The annealing step both leads to the organometallic decomposition and full solvent evaporation. During this evaporation, the Ni NPs are pulled to the edges by a coffee ring effect. In the present study, an in-depth analysis reveals a new feature: the very thin parallel guidelines observed on each side of the lines (see Fig. 5 AFM image for instance).

J. Ye and C.V. Thompson (Ye \& Thompson, 2010) - who investigated solid-state dewetting of singlecrystal nickel thin films on $\mathrm{MgO}$ surface -, reported that in a patterned film, there is a large gradient in surface curvature around the edge of the film, and atoms therefore diffuse from the edge to the top surface of the film. Thanks to this process the edge of the film retracts and a thickening rim develops near the edge of the film (Fig. 7A). The effect is visible on Fig. 7B in the case of the Ni lines formed during our experiments on silicon substrate. The annealing temperature, although considered as low (below $150{ }^{\circ} \mathrm{C}$ ), enables the diffusion of Ni NPs to form the thicker borders observed by AFM. 

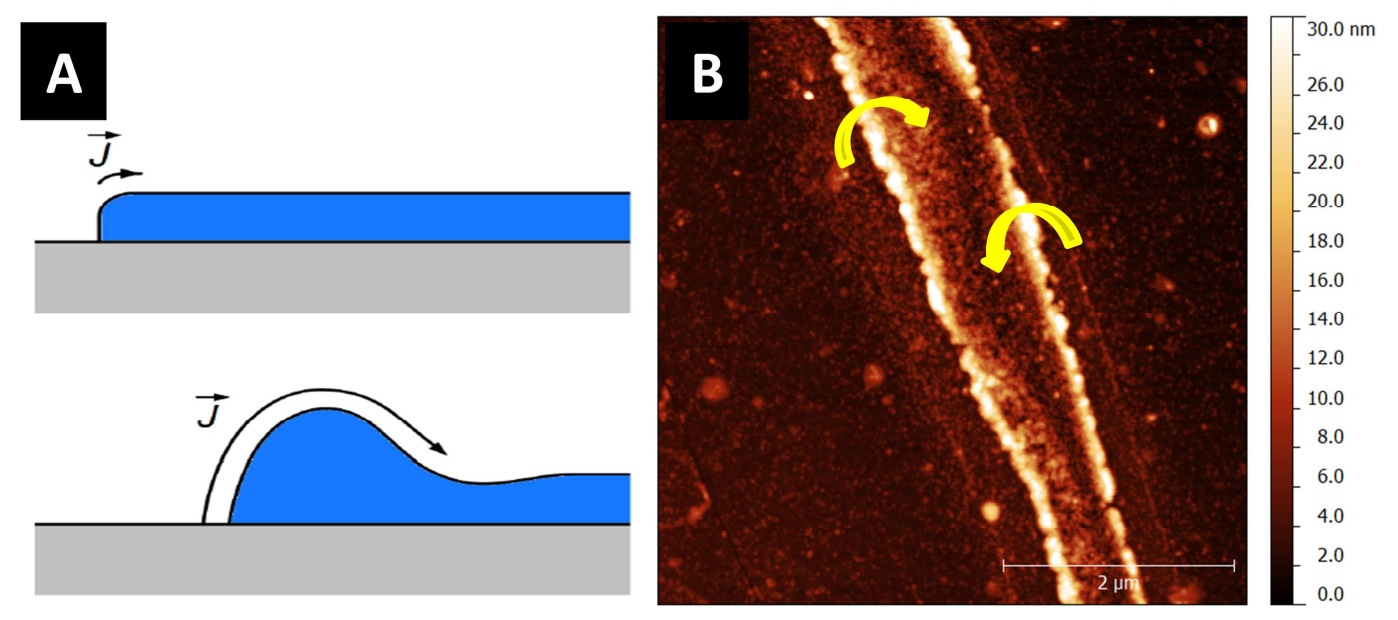

Figure 7: Schema of rim thickening (A) (Ye \& Thompson, 2010); thickening rim developed near the edge of the Ni-line observed by AFM (B)

\section{Zigzag shape}

The development of zigzag morphology begins with the nucleation and growth of holes in thin film spin coated on silicon substrate. The holes have specific shape depending from the drying process during spin coating and thermal decomposition of metallic precursor. The two typical in-plane directions are marked with blue and green lines on Fig. 8. These two directions form an angle of $120^{\circ}(\pi / 3)$ which is typical of closed-packed structure. Noticeably, spherical colloids spontaneously form hexagonal packing upon drying on a flat surface and results in the formation of edges or line defects with $\pi / 3$ angles between them. Ni NPs formed in situ could diffuse and form such an assembly, although we could not observe such an ordered structure between the NPs.
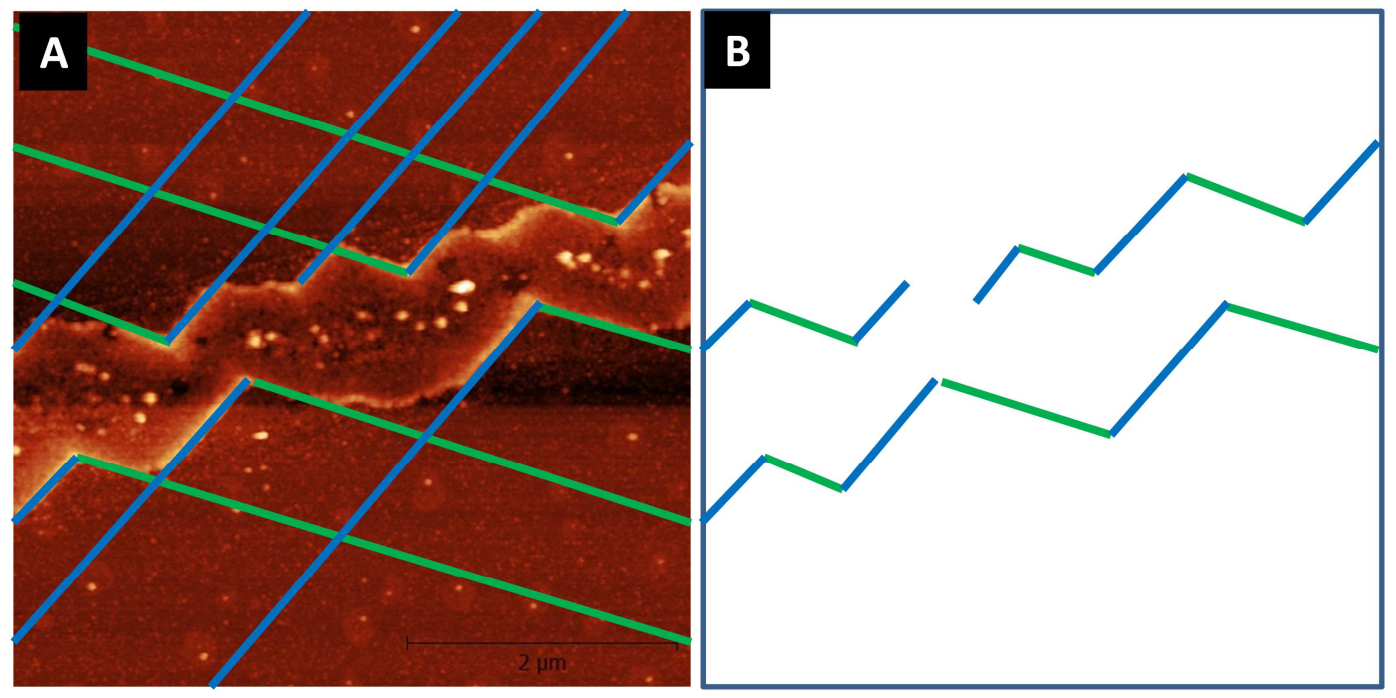
Figure 8: Major directions of zigzag line shape formation on an AFM image (A) and guidelines (B)

On Fig. 8B, the blue and green lines are closely parallel on both side of the formed nickel nanoribbon. Therefore, another explanation could prevail concerning the zigzag origin. On single crystals, the surface energy anisotropy affects the edge retraction rate, and leads to different retraction rates for edges aligned with different in-plane directions. The rate of edge retraction can depend also from the substrate. In the early stage of dewetting of single-crystal films, holes grow with specific in-plane facetted shapes that depend on the crystallographic orientations of the films and annealing parameters (Ye \& Thompson, 2011).

Based on the literature (Ye \& Thompson, 2011), anisotropic surface energy and surface diffusion lead to edge retraction velocities, the dominating surface energy anisotropy lead to sharp cusps (zigzag shape) observed at the edges of formed nanowires. It should be taken into consideration that other theories (Deegan, 2000) can also describe the zigzag shape formation on the basis of competition between dewetting and pinning forces. It may be well possible that a saw-toothed front is caused by a piecewise transition to a double layer film. First, a few points along the contact line begin to produce two layers. As the contact line retreats, the segments producing double layers extend along the contact line, leaving a zigzag-shaped deposit.

\subsection{Co and NiFe lines}

We have investigated next to $\mathrm{Ni}$ nanowires also $\mathrm{Co}$, Ni-Fe bimetallic line-formation using $\mathrm{Co}_{2}(\mathrm{CO})_{8}$ and $\mathrm{Fe}(\mathrm{CO})_{5}$ as metallic precursors. Bimetallic nanowire formation can open a larger horizon and allows tuning the magnetic properties.

$\mathrm{Co}_{2}(\mathrm{CO})_{8}$ has been widely used as a precursor for cobalt nanoparticles in solution reaction yielding spherical (Sun \& Murray, 1999) (Yang, et al., 2004) or disk shape (Puntes, V. F.; Zanchet, D.; Erdonmez, C. K.; Alivisatos, A. P., 2002) particles in a temperature range of $180-220^{\circ} \mathrm{C}$. On the other hand, $\mathrm{Co}_{2}(\mathrm{CO})_{8}$ has been rarely used in dry processes and only several studies report on MOCVD processes under low pressure at temperatures between 70 and $130{ }^{\circ} \mathrm{C}$ (Lee, et al., 2006).

Spin coating and thermal decomposition of $\mathrm{Co}_{2}(\mathrm{CO})_{8}$ is therefore performed at $80^{\circ} \mathrm{C}$, as discussed in the case of $\mathrm{Ni}(\mathrm{COD})_{2}$. Metallic lines can be observed only for very high speed (10,000 rpm). Co lines formed on glass substrate are presented on Fig. 9A, The average thickness of the lines is $1.1 \pm 0.3 \mu \mathrm{m}$ with a length of several hundreds of $\mu \mathrm{m}$. The formation of wires of particles seems to be driven by the dewetting of the precursor solution, implying the formation of lines of dry precursors as suggested with $\mathrm{Ni}(\mathrm{COD})_{2}$. The wires display a ferromagnetic behaviour at $\mathrm{T}=2 \mathrm{~K}$ (Fig. 9B) with a weak hysteresis $\left(\mu_{0} \mathrm{H}_{\mathrm{C}}=1.2\right.$ 
$\mathrm{mT})$. However, using different spin coating and annealing parameters, no zigzag line formation is observed for any of the samples using carbonyl compounds of Co. The thermal decomposition of $\mathrm{Co}_{2}(\mathrm{CO})_{8}$ yields gaseous $\mathrm{CO}$ as a by-product (Puntes, et al., 2001), while the decomposition of $\mathrm{Ni}(\mathrm{COD})_{2}$ at $80^{\circ} \mathrm{C}$ yields liquid cyclooctadiene. Therefore, the nucleation of Co occurs on a dry substrate while $\mathrm{Ni}$ nucleation occurs on a wet substrate, resulting in a larger diffusion of Ni. Hence, the use of carbonyl precursors should open a way to bimetallic systems.
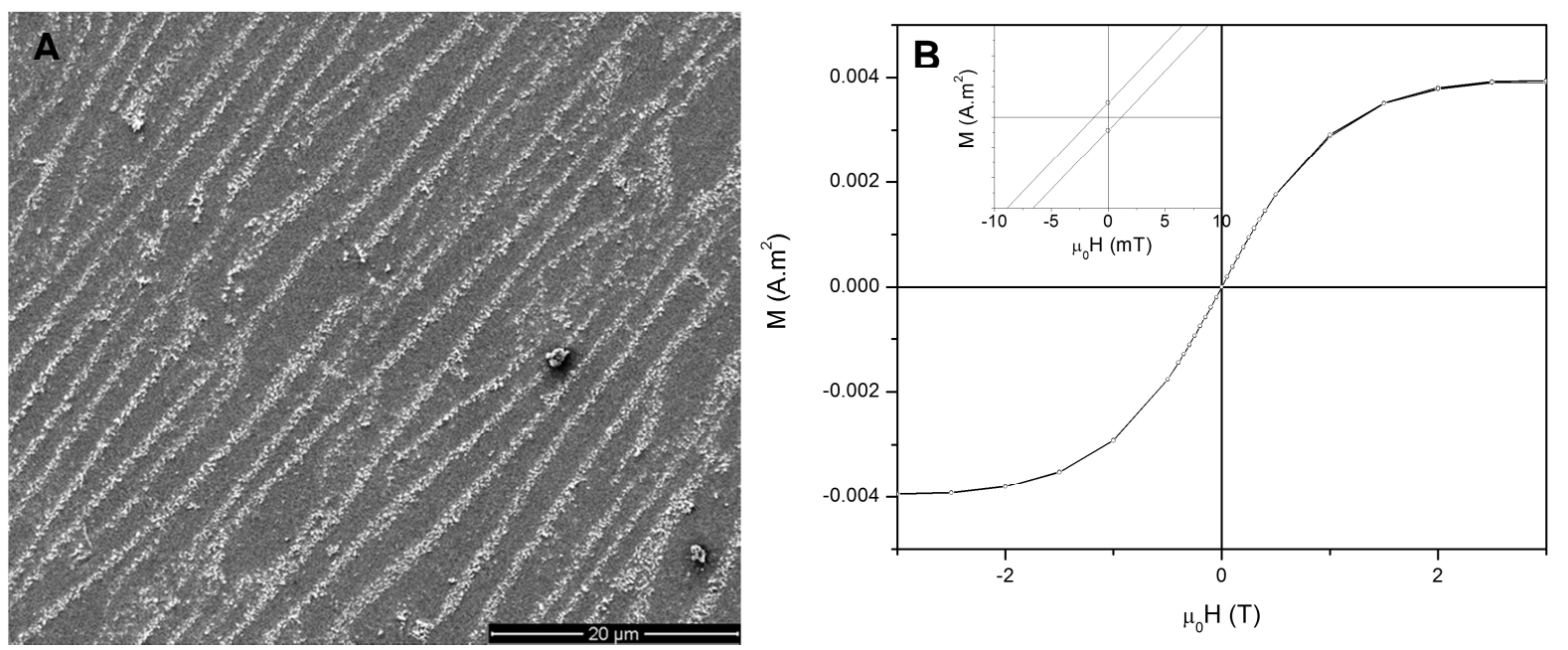

Figure 9: Co lines on glass substrate: SEM micrograph (A) and Magnetic measurement at 2K (inset: magnification of the hysteresis) (B)

As an example, $\mathrm{Ni}(\mathrm{COD})_{2}$ and $\mathrm{Fe}(\mathrm{CO})_{5}$ organometallic precursors should yield bimetallic magnetic wires with interesting magnetic properties (Fodor, et al., 2002). $\mathrm{Fe}(\mathrm{CO})_{5}$ remains the most readily available precursors for Fe and successfully yields Fe nanoparticles in solution (Park, et al., 2000) (Lacroix, et al., 2011) or as MOCVD precursor (Senocq, et al., 2006). Nevertheless, metal carbonyl precursors should be used cautiously with $\mathrm{Ni}(\mathrm{COD})_{2}$ since they can readily react at room temperature yielding a black precipitate. Therefore, $\mathrm{Ni}(\mathrm{COD})_{2}$ and $\mathrm{Fe}(\mathrm{CO})_{5}$ are deposited in two successive steps to direct the reaction on the substrate. The spin coating is performed in the same conditions at $4000 \mathrm{rpm}$ with an annealing step after the process.

Parallel lines of metallic nanoparticles can be observed by AFM (Fig. 10A-B). The lines follow a define pattern with pairs of parallel lines, with gaps of $0.6 \pm 0.1 \mu \mathrm{m}$ between them and gaps of $2.2 \pm 0.2 \mu \mathrm{m}$ from one pair to the other (center to center). The particles are around $5 \mathrm{~nm}$ thick and each narrow line is only one particle wide. Each band seems to result from the phenomenon already observed with $\mathrm{Ni}(\mathrm{COD})_{2}$ alone. The parallel lines consist of the edges of elongated droplets after drying. The heating step is now replaced by the deposition of $\mathrm{Fe}(\mathrm{CO})_{5}$ which spontaneously decompose the nickel precursor, yielding 
metallic nanoparticles. The composition and magnetic properties of these wires are under investigation and require advanced techniques to account for the very small amount of material.
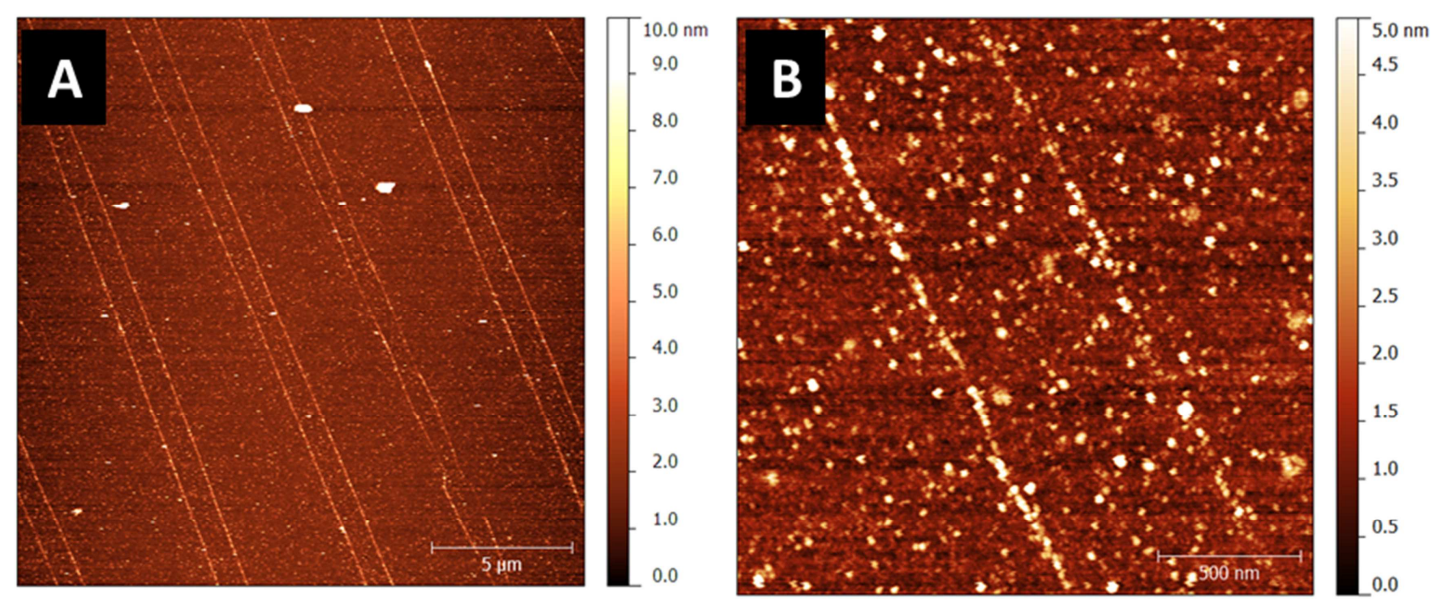

Figure 10: AFM images of bimetallic nanowire formation of Ni-Fe system, spin coating parameters $\mathrm{Ni}$ $4000 \mathrm{rpm}$ and $\mathrm{Fe} 4000 \mathrm{rpm}(\mathrm{A})$ and (B)

In the presence of $\mathrm{Fe}(\mathrm{CO})_{5}$, Ni nucleates very fast even at room temperature, yielding small particles (5$10 \mathrm{~nm}$ ) which dispersion dries according to a standard coffee effect. This fast nucleation before any evaporation explains the formation of shadow lines exactly on the edges of the original elongated droplets (Fig. 10). Based on these results, we can conclude that the zigzag line formation depends also on the nature of the organometallic precursor and especially the decomposition kinetics, next to spin coating and annealing parameters. Using different spin coating and annealing parameters, no zigzag line formation is observed for any of the samples using carbonyl compounds of Co or Fe. In the case of $\mathrm{Ni}(\mathrm{COD})_{2}$, the evaporation process fortunately matches the kinetics of decomposition and allows us observing formation of zigzag lines.

\section{CONCLUSION}

Organometallic liquid deposition of $\mathrm{Ni}$ can be directed by spin coating with two main experimental parameters: temperature which controls the $\mathrm{Ni}$ "contrast" between lines and speed which control the line shape. The origin of the line formation lies in the evaporation process of the wet thin film under centrifugal force. The specific shape observed could be explained by an anisotropic coffee ring effect, resulting in 1D nanostructures. 
Low temperature $\left(80^{\circ} \mathrm{C}\right)$ process allows for the slow diffusion of $\mathrm{Ni}$ clusters which creates unique zigzag shape Ni nanowires in a reproducible way. Ni clusters self-assemble and form sharp angles upon drying which we assume as a result of competition between dewetting and pinning forces. The line formation occurs for other materials including $\mathrm{Co}$ and $\mathrm{NiFe}$, while the zigzag structure is specific to the $\mathrm{Ni}(\mathrm{COD})_{2}$ precursor deposited under specific parameters (speed of spin coating, temperature of thermal decomposition).

\section{AUTHOR INFORMATION}

Corresponding Author

*E-mail: David.zitoun@biu.ac.il

\section{ACKNOWLEDGMENTS}

The authors thank Dr Olga Girshevitz for assistance with AFM.

\section{REFERENCES}

Choi, S., S., S., Pisano, A. P. \& I., Z. T., 2010. Coffee-Ring Effect-Based Three Dimensional Patterning of Micro/Nanoparticle Assembly with a Single Droplet. Langmuir, Volume 26, p. 11690-11698.

Deegan, R. D., 2000. Pattern formation in drying drops. PHYSICAL REVIEW E, Volume 61, pp. 475-485.

Deegan, R. D. et al., 1997. Capillary flowasthe cause of ring stains from dried liquid drops. Nature, Volume 389, pp. 827-829.

Felici, R.; Jeutter, N. M.; Mussi, V.; Buatier de Mongeot, F.; Boragno, C.; Valbusa, U.; Toma, A.; Zhang, Y. W.; Rau, C.; Robinson, I. K., 2007. In situ study of the dewetting behavior of Nifilms on oxidized Si(001) by GISAXS. Surface Science, Volume 601, pp. 4526-4530.

Fodor, P. S., Tsoi, G. M. \& Wenger, L. E., 2002. Fabrication and characterization of Co1-xFex alloy nanowires. J. Appl. Phys., Volume 91, pp. 8186-8188.

$\mathrm{He}$, X. et al., 2007. Synthesis and characterization of silver nanowires with zigzag morphology in N,N-dimethylformamide. Journal of Solid State Chemistry, Volume 180, p. 2262-2267.

Hyun, D. C. et al., 2011. Ordered Zigzag Stripes of Polymer Gel/Metal Nanoparticle Composites for Highly Stretchable Conductive Electrodes. Adv. Mater, Volume 23, p. 2946-2950.

Kim, H. et al., 2008. Spatial Control of Coherent Anti-Stokes Emission with Height-Modulated Gold Zig-Zag Nanowires. Nano Lett., Volume 8, pp. 2373-2377.

Lacroix, L.-M.et al., 2011. Stable Single-Crystalline Body Centered Cubic Fe Nanoparticles.

Nano lett., Volume 11, p. 1641-1645.

Lee, J. et al., 2006. Highly Conformal Deposition of Pure Co Films by MOCVD Using

Co2 ( CO ) 8 as a Precursor. J. Electrochem. Soc., Volume 153, p. G539-G542.

Müller, C. M. \& Spolenak, R., 2010. Microstructure evolution during dewetting in thin Au films. Acta Materialia, Volume 58, p. 6035-6045. 
Park, S. et al., 2000. Synthesis and Magnetic Studies of Uniform Iron. J. Am. Chem. Soc., Volume 122, p. 8581-8582.

Petersen, J. \& Mayr, S. G., 2008. Dewetting of Ni and NiAg solid thin films and formation of nanowires on ripple patterned substrates. Journal of Applied Physics, Volume 103, pp. 023520$1-8$.

Puntes, V. F.; Zanchet, D.; Erdonmez, C. K.; Alivisatos, A. P., 2002. Synthesis of hcp-Co Nanodisks. J. Am. Chem. Soc., Volume 124, p. 12874-12880.

Puntes, V. F., M., K. K. \& Alivisatos, A. P., 2001. Colloidal Nanocrystal Shape and Size Control: The Case of Cobalt. Science, Volume 291, pp. 2115-2117.

Senocq, F. et al., 2006. Iron Thin Films from Fe ( CO ) 5 and FeCp2/H2O under Atmospheric Pressure. J. Electrochem. Soc., Volume 153, p. G1025-G1031.

Shviro, M.; Zitoun, D., 2012. Low temperature, template-free route to nickel thin films.

Nanoscale, Volume 4, pp. 762-767.

Shviro, M. \& Zitoun, D., 2013. Nickel nanocrystals: fast synthesis of cubes, pyramids and tetrapods. RSC Adv., Volume 3, pp. 1380-1387.

Sikora, E. \& Macdonal, D., 2002. Nature of the passive film on nickel. Electrochimica Acta, Volume 48, pp. 69-77.

Sun, S. \& Murray, C. B. J., 1999. Synthesis of monodisperse cobalt nanocrystals and their assembly into magnetic superlattices. Appl. Phys., Volume 85, pp. 4325-4330.

Xia, Y., Qin, D. \& Yin, Y., 2001. Surface patterning and its application in wetting dewetting studies. Current Opinion in Colloid \& Interface Science, Volume 6, pp. 54-64.

Yang, H. T. et al., 2004. Stable cobalt nanoparticles passivated with oleic acid and triphenylphosphine. Nanotechnology, Volume 15, p. 70-74.

Ye, J. \& Thompson, C. V., 2010. Mechanisms of complex morphological evolution during solidstate dewetting of single-crystal nickel thin films. Appl. Phys. Lett., Volume 97, pp. 071904-1-3. Ye, J. \& Thompson, C. V., 2011. Anisotropic edge retraction and hole growth during solid-state dewetting of single crystal nickel thin films. Acta Materialia, Volume 59, p. 582-589.

Ye, J. \& Thompson, C. V., 2011. Templated Solid-State Dewetting to Controllably Produce Complex Patterns. Adv. Mater, Volume 23, p. 1567-1571.

Yu, C. et al., 2003. Fabrication and physical properties of permalloy nano-size wires. Physica B, Volume 327, pp. 247-252.

Zhang, J. et al., 2002. Self-Assembled Nanostructures. s.1.:Springer. 